Aims. Clinical Simulation sessions were started in April 2020 to supplement reduced patient contact for medical students at the University of Sheffield due to COVID-19 restrictions. These were run by Foundation Trainees in psychiatry with supervision and oversight from a senior psychiatrist. This study aims to review current literature on remote teaching as a learning resource and will evaluate the effectiveness of clinical simulation as an alternative to patient contact, with the focus being on improving students' confidence as well as developing clinical interview skills.

Method. Feedback surveys were developed, focussing on confidence undertaking difficult aspects of psychiatric interviews, and distributed amongst two cohorts of medical students at the University of Sheffield. One cohort completed their face-to-face psychiatry placement in full pre-COVID, the other undertook placements consisting of virtual simulation sessions alongside reduced patient contact. Responses were collected online over 6 weeks between February and March 2021. As two medical students who completed face-to-face psychiatry placement prior to the pandemic, we have additionally submitted personal reflections as a comparator to current student experiences.

Result. A total of 8 students in the clinical simulation cohort, and a total of 13 students from the face-to-face teaching cohort completed the questionnaire. $62.5 \%$ of students that responded were female and the remaining percentage identified as male. Students in the face-to-face cohort reported being more confident in 6 out of 7 aspects of our feedback surveys determining confidence undertaking clinical interview skills in comparison to the virtual simulation cohort. Students attended varying numbers of simulation sessions and ultimately the main restrictions and barriers to the simulation teaching reported by students are the time constraints during the sessions, and unstable internet connection. Conclusion. Overall confidence levels in medical students are undoubtedly higher in students that completed full face-to-face placements in comparison to those with combined teaching. Based on student responses and review of current literature, clinical simulation appears to serve as a useful adjunct to students with reduced face-to-face contact in psychiatry, particularly for increasing confidence when interviewing more challenging patients. Immediate facilitator feedback and exposure to more difficult patient scenarios seem to be the most beneficial aspects. We would not advocate it as an exclusive form of teaching for medical students, but it may be a useful resource post-pandemic for providing students with extra learning opportunities, specifically targeted at developing confidence and skills in more difficult situations which will hopefully benefit them in their later careers.

\section{The haematological side effects of clozapine: literature review and meta-analysis}

Tariq Munshi ${ }^{1 \star}$, Farooq Naeem², Mohammed Ayub $^{3}$, Saeed Farooq ${ }^{4}$, Davit Khachatryan ${ }^{5}$, Selim Asmer ${ }^{3}$, Peter Wang ${ }^{5}$, Shahista Premji ${ }^{5}$, Christine Van Winssen ${ }^{5}$, Felix Lau ${ }^{5}$ and Jonathan Fairbairn ${ }^{5}$

${ }^{1}$ St Michael's Hospital, University of Toronto; ${ }^{2}$ Canadian Addictions and Mental Health, University of Toronto; ${ }^{3}$ Queens University;

${ }^{4}$ Keele University and ${ }^{5}$ Queen's University

${ }^{*}$ Corresponding author.

doi: 10.1192/bjo.2021.729

Aims. This review and meta-analysis aim to estimate the cumulative incidence of clozapine induced agranulocytosis and leukopenia the impact of the associated factors such as dose of clozapine, duration of follow-up, gender and race on the cumulative incidence.

Background. Clozapine is the only medication licensed for treatment-resistant schizophrenia. There has been a renewed interest in the role of Clozapine in the treatment of Schizophrenia based on strong evidence that favours its efficacy and safety. Despite the evidence that Clozapine has superior efficacy and has been recommended for treatment-resistant cases by the national guidelines, the drug is underutilised.

Method. We included all studies in which clozapine was used for a psychotic illness. We included studies which provided data on two primary indices; Leucopenia or agranulocytosis and neutropenia; defined according to the cut off used by CPMS for total WBC and neutrophil count. Additionally we included studies reporting another blood dyscrasia or death due to agranulocytosis. Studies were identified by searching AMED, BIOSIS, CINAHL, EMBASE, MEDLINE, PsycINFO, PubMed, and registries of Clinical Trials and their monthly updates, hand searches, gray literature, and conference proceedings from the first available date until 2nd February, 2015. The search was updated on 15th March, 2017. The Protocol was initiated and then registered with PROSPERO International prospective register of systematic reviews University of York, Centre for Reviews and Dissemination.

Result. The cumulative incidence of the agranulocytosis in all studies was $00.32 \%$ (CI 00.1-0.63). The cumulative incidence in all studies for different types of blood dyscrasia were following: leucopenia $00.96 \%$ (CI $0.39-1.70$ ), neutropenia $2.93 \%$ (CI 1.49-4.72), other blood dyscrasias $4.64 \%$ (CI 2.34-7.52) and any blood dyscrasia was 2.23 (CI 1.46-3.12).

Conclusion. The limitations of this review are mainly due to the nature of evidence from the included studies. We adopted a broad inclusion criteria to include all the available evidence. Number of patients started on Clozapine may be withdrawn from the Clozapine on the earliest signs of blood dyscrasias since the introduction of Clozapine monitoring services. This means that the true incidence of agranulocytosis and neutropenia may be higher and this may be a major bias in finding the true incidence of Clozapine induced neutropenia.

\section{Dissection of neuroinflammation in schizophrenia}

Fizah Muratib*, Yuya Mizuno, Ines Carreira Figueiredo, Oliver Howes and Tiago Reis Marques

IoPPN King's College London

${ }^{*}$ Corresponding author.

\section{doi: 10.1192/bjo.2021.730}

Aims. Schizophrenia is notoriously becoming one of the world's most debilitating mental disorders, affecting 1 in 100 people. There is increasing evidence that neuroinflammation plays a part in the pathogenesis of schizophrenia and other psychotic disorders; microglial activity acting as a marker for neuroinflammatory reactions in the brain. Furthermore, cannabis is an illicit substance that also evokes a similar response in the neuroimmune activity. This project explores how cannabis exposure influences an elevation in neuroinflammatory responses through TSPO levels, and whether this information can help us determine if cannabis use and increased TSPO levels can be associated with a risk factor for developing psychosis.

Method. 55 participants (36 males and 19 females) were recruited from the community by the IRIS (Inflammatory Reaction in Schizophrenia) team at the IoPPN, King's College London, from which 34 patients with a diagnosis of schizophrenia and 21 healthy controls took part in the study. The eligible participants underwent clinical assessments and PET scanning, from which cannabis use history and PET data were collected. Participant neuroinflammatory levels are represented by [18F]DPA-714 volume and different regions of grey matter in the brain were analysed through multivariate analyses, the confounding variables being age and TSPO genotype. 
Result. A statistically significant association is shown between participants who have had exposure to cannabis and participants who have not had any exposure in their lifetime. The differences across the prioritised brain regions of interest were robust, the association appearing more apparent and statistically significant in the total $(\mathrm{p}=.00)$ and temporal grey matter $(\mathrm{p}=.00)$ regions of the brain. This may suggest that cannabis exposure influences the [18F]DPA-714 VT in the significant regions of interest. However, a negative association is seen with current use, the quantity of use, and the frequency of use.

Conclusion. The initial findings for cannabis exposure show us a positive association with increased TSPO levels, however, limitations must be taken into account. Although we cannot readily establish that elevated TSPO levels in cannabis users can presently act as a risk factor marker for developing psychosis from this particular study, we can utilise this data to continue our research in disclosing a new system to predict the occurrence of psychosis.

\section{The mental health of elite rugby players (a literature review)}

Kirk Musgrave

TEWV NHS Foundation Trust

doi: $10.1192 /$ bjo.2021.731

Aims. Players are Rugby's key asset, what recent research has been conducted into the Mental Health of rugby players/former players?

Method. Initially a Literature Search using HDAS, Ebsco, Researchgate and Googlescholar followed by a Literature Review of relevant articles.

Result. A significantly higher prevalence of anxiety and depressive symptoms in Professional rugby players (compared to the general population) is something that authors agree on. This review considers some of the rugby specific variables not limited to injuries (including concussion), retirement from the sport and finally alcohol abuse.

In 2014, Sullivan looked at the role of potential mediators between concussion and later life depression. Sullivan suggested that the effects of concussion on later life depression may be directly neurological.

Chronic Traumatic Encephalopathy (CTE) is a neurodegeneration which is only definitively diagnosed by post-mortem examination of brain tissue at this time. Today, Chronic Traumatic Encephalopathy is a very controversial subject, for every piece of research which claims to prove CTE, there is another piece of research apparently disproving it.

Alcohol Misuse - Whilst it is well known in general adult psychiatry that alcohol has a significant negative impact on depression and anxiety in the general population, this review summarises findings from research into alcohol misuse in elite rugby players.

Conclusion. In addition to personal variables (which include personality, perfectionism, ability to cope with stress, optimism, pessimism, ability to utilise mental skills, burnout and career satisfaction) there are rugby specific variables which are not limited to injuries, retirement from the sport and finally alcohol abuse.As mentioned in the paragraph on depression and anxiety, numerous recently published authors agree that a significantly higher prevalence of anxiety and depressive symptoms are seen in Professional rugby players (compared to the general population).

As alcohol misuse has already been researched, there would seem to be an opportunity for future research into the extent of illicit drug use by elite rugby players and potentially the effect of illicit drug use on depressive symptoms and anxiety. As mentioned in the paragraph on depression and anxiety, numerous recently published authors agree that a significantly higher prevalence of anxiety and depressive symptoms are seen in Professional rugby players (compared to the general population).

Finally, given the limited recent published literature on suicide in elite rugby players and former elite rugby players, a significant research gap exists in this particular field.

Using electronic clinical records to investigate service use and in-patient care of adults with intellectual disability and/or autism spectrum disorder

Jennifer Mutch ${ }^{1 \star}$, Rory Sheehan ${ }^{2}$, Louise Marston ${ }^{2}$, Nomi Werbeloff ${ }^{2}$, David Osborn ${ }^{2}$ and Angela Hassiotis ${ }^{2}$

${ }^{1}$ NHS Lothian Learning Disability Service and ${ }^{2}$ University College London

${ }^{\star}$ Corresponding author.

doi: $10.1192 /$ bjo.2021.732

Aims. To describe characteristics of adults with intellectual disability (ID) and/or autism spectrum disorder (ASD) accessing care in one mental health Trust.

To explore factors associated with in-patient admission/risk of re-admission within 12 months of discharge.

Background. There is concern that adults with intellectual disability and those with autism spectrum disorder are frequently admitted to mental health hospitals. The evidence from NHS datasets suggests that this remains a significant issue and is associated with personal, social and economic costs.

Method. Adults ( $\geq 18$ years) with ICD-10 diagnosis of "mental retardation" and/or autism who had accessed care in the Camden and Islington Foundation Trust were identified using the Clinical Record Interactive Search (CRIS). The identification process was validated through cross checking of free text in the electronic clinical notes. We compared demographic and clinical characteristics and service use, including length of admission, of 315 individuals with ASD and 339 with ID (with or without ASD). Logistic regression was used to explore factors associated with in-patient admission and re-admission within 12 months of discharge.

Result. A greater proportion of adults with ID (with or without ASD) had a diagnosis of psychosis, substance misuse, or dementia whereas diagnosis of anxiety disorder was greater in those with ASD. Antipsychotics and other psychotropics were twice as likely to be prescribed for the ID \pm group. Admission to psychiatric in-patient care was greater in those with ID \pm ASD (adjusted OR 4.00, 95\% confidence interval (CI) 2.41-6.63), men (aOR $2.28,95 \% \mathrm{CI} 1.39-3.75$ ), younger adults (aOR 0.98, 95\%CI 0.97-1.00), and in those with a diagnosis of schizophrenia spectrum disorder (aOR 5.08, 95\%CI 3.00-8.61), affective disorder (aOR 2.23, 95\%CI 1.29-3.83), personality disorder (aOR 1.94, 95\%CI 1.02-3.68), and record of previous inpatient admission (aOR 2.18, 95\%CI 1.17-4.05). Having ASD alone was associated with a greater risk of re-admission within one year of discharge, although this difference was not statistically significant (aOR $0.70,95 \%$ CI 0.32-1.52). Comorbid diagnoses of affective disorder or personality disorder were the only significant associations with re-admission (aOR 3.11, 95\%CI 1.34-7.23 and aOR 8.28, 95\%CI 2.85-24.04, respectively).

Conclusion. These findings provide the first longitudinal investigation into the acute care pathway for adults with ID and/or ASD in the NHS. Replication in other trusts is now needed to inform "at risk of admission" registers and guide targeted interventions to prevent admission. 